\title{
Retrocausal Quantum Mechanics: Maudlin's Challenge Revisited
}

\author{
August 23, 2013
}

\begin{abstract}
In 1994, Maudlin proposed an objection to retrocausal approaches to quantum mechanics in general, and to the transactional interpretation (TI) in particular, involving an absorber that changes location depending on the trajectory of the particle. Maudlin considered this objection fatal. However, the TI did not die; rather, a number of responses were developed, some attempting to accommodate Maudlin's example within the existing TI, and others modifying the TI. I argue that none of these responses is fully adequate. The reason, I submit, is that there are two aspects to Maudlin's objection; the more readily soluble aspect has received all the attention, but the more problematic aspect has gone unnoticed. I consider the prospects for developing a successful retrocausal quantum theory in light of this second aspect of the objection.
\end{abstract}

\section{Retrocausal quantum mechanics}

Cramer's (1986) transactional interpretation (TI) begins from the observation that relativistic versions of the quantum mechanical equation of motion admit both retarded solutions - waves propagating towards the future - and advanced solutions - waves propagating towards the past. The advanced solutions are usually discarded as unrealistic, but Cramer suggests incorporating them into an interpretation of quantum mechanics.

As a simple illustration, let us apply the TI to the experiment shown in figure 1. A particle is emitted by a source $\mathrm{S}$ at time $t_{0}$, passes through 


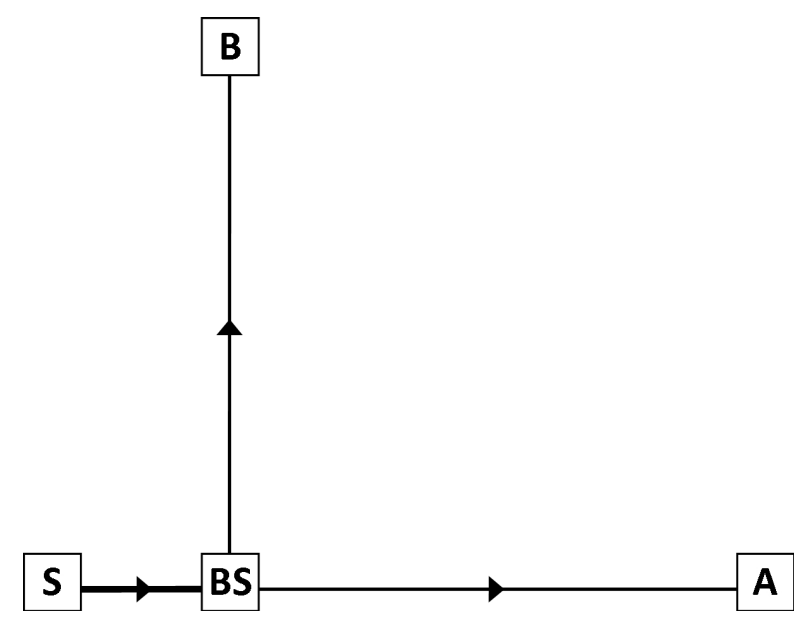

Figure 1: A simple experiment.

a symmetric beam-splitter BS, and is absorbed either by absorber A or by absorber $\mathrm{B}$ at time $t_{1}$. According to the TI, at $t_{0}$ the source emits a retarded wave - the offer wave - in the direction of the beam splitter, where it is split into two equal-amplitude components that travel to $\mathrm{A}$ and $\mathrm{B}$ respectively. The arrival of an offer wave at $\mathrm{A}$ at $t_{1}$ triggers the emission of an advanced wave of the same amplitude and phase - the confirmation wave-travelling back towards the source at $t_{0}$. The same process occurs at B. So there are now two equal-amplitude offer-confirmation pairs between the emitter and the absorbers, one going to $\mathrm{A}$ and one to $\mathrm{B}$. These are regarded as competing possible transactions; one of them becomes actual and the other does not. This is a fundamentally indeterministic process, and the chance of each possible transaction becoming actual is given by the product of the offer wave amplitude and the confirmation wave amplitude. In this case, each completed transaction has a probability of $1 / 2$. If the completed transaction is between the source and absorber A, then the particle goes to A, and similarly for B.

The incorporation of advanced waves is mathematically satisfying because it avoids throwing away half the solutions to the equation of motion. But it also allows a uniform treatment of several puzzling quantum phenomena, treated at length in Cramer (1986). In particular, since the advanced confirmation wave can transmit information about the measurements that are to be performed on a particle back in time to the particle source, no non- 
local influences are required to explain the results of EPR-Bell experiments (1986, 667). Several other interpretations of quantum mechanics have been developed to exploit this retrocausal strategy, including those of Price (1994), Sutherland (2008) and Wharton (2010).

But the TI and other retrocausal approaches are challenged by Maudlin (1994). Maudlin offers two arguments, an initial objection is aimed at the particular theoretical structure of the TI, and a more general challenge aimed at retrocausal theories in general. I will take them in turn.

\section{Pseudotime and explanation}

Maudlin's initial objection concerns the status of the pseudotime narrativethe TI story about how the transaction is established. On the surface, at least, the pseudotime narrative provides the central explanatory mechanism of the TI; offer waves are sent forwards in time from the particle source to the potential absorbers, and then each absorber returns a confirmation wave backwards in time to the source, and then one offer-confirmation pair at random becomes the completed transaction. Maudlin regards this explanatory mechanism as problematic. The locution "and then" in the pseudotime narrative cannot be understood temporally, because the events connected by this locution zigzag back and forth in time. Neither can it be understood causally, because only the completed transaction actually exists, so the offerconfirmation process that precedes it in the pseudotime narrative never actually occurs. But then how should we understand it? Cramer himself backs away from taking the pseudotime narrative literally, describing it as "only a semantic convenience", a useful fiction $(1986,663)$. Maudlin's first objection to the TI is that Cramer cannot afford to make such a concession. Taken literally, the pseudotime narrative is incoherent, but since "all of the seeming illumination provided by the account depends on the pseudotime narrative", the TI without the narrative fails as an explanatory theory $(1994,198)$.

But is Maudlin right that the pseudotime narrative provides all the illumination of the TI? This seems unfair, as immediately following Cramer's concession that the pseudotime narrative is a useful fiction he provides an alternative way of understanding the TI that makes no appeal to the pseudotime narrative whatsoever: "An equally valid interpretation of the process is that a four-vector standing wave has been established between emitter and absorber" $(1986,663)$. That is, given that nature contains both advanced 
and retarded waves, and given that the arrival of a retarded offer wave triggers the emission of an advanced confirmation wave and vice versa, what we should expect to see is a standing wave between the source and an absorber composed of an advanced and a retarded component. After all, anything other than a standing wave would be inconsistent, ascribing distinct wave amplitudes successively to one and the same spacetime point. Hence one can bypass the pseudotime narrative, and simply appeal to consistency as an explanation of the completed transactions one sees. The advanced and retarded waves of the TI have a crucial role in this explanation, as components of the standing wave, but this role is not the one they play in the pseudotime narrative.

However, the standing wave explanation does not yield the probabilities with which we see each of the possible completed transactions, so in this sense the explanation is incomplete. But quantum probabilities are rarely explained; except for a controversial research program in many-worlds quantum mechanics (Deutsch 1999), quantum theories typically stipulate a rule for ascribing probabilities to outcomes rather than explaining that rule. So the TI is in good company here. Perhaps the easiest way to state the rule is in terms of the pseudotime narrative; if an offer wave from the source were to produce confirmation waves from every absorber, the probability of a transaction is the product of the amplitudes of the offer and confirmation waves connecting the source to the relevant absorber. But this appeal to a fragment of the pseudotime narrative is entirely instrumental; it simply provides a straightforward mathematical recipe for assigning probabilities to trajectories.

So Maudlin is wrong that the pseudotime narrative is essential to the TI; one can eliminate the pseudotime narrative as a causal explanation without sacrificing the illumination that the TI approach provides. But Maudlin is also remiss, I think, in implying that the pseudotime narrative can provide no illumination because it is an inconsistent fiction. Fictions, even inconsistent ones, can provide a kind of illumination. In particular, the pseudotime narrative provides a way to come to the realization that only a standing wave solution is possible, even if it cannot be the causal mechanism by which those standing waves come about. So it seems entirely apt for Cramer to describe the pseudotime narrative as a semantic convenience and a "pedagogical convention" (1986, 661); nothing like the pseudotime narrative could actually occur as a causal process, but nevertheless this fiction is a good way for students to discover for themselves why standing wave solutions are what 


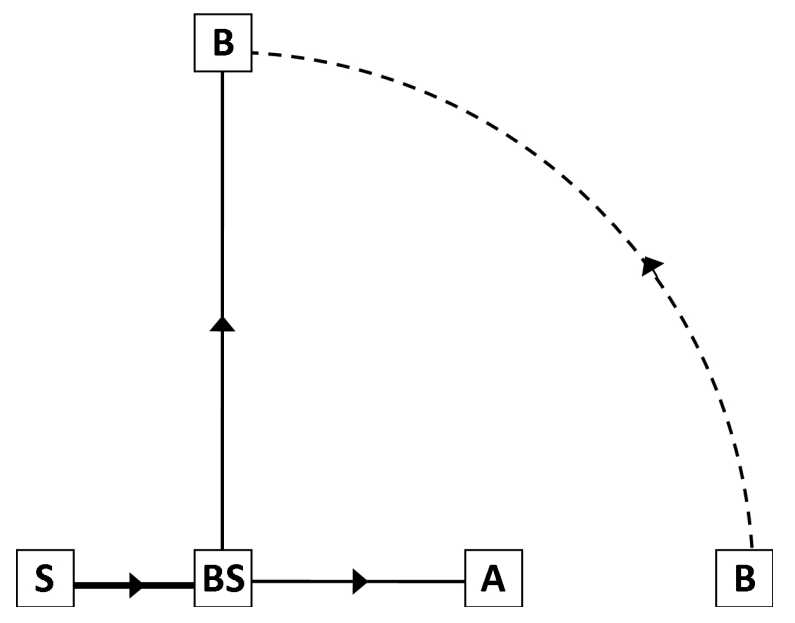

Figure 2: Maudlin's experiment.

we observe, and in this way the fiction provides illumination.

So Maudlin's initial complaint against the TI is, I think, misplaced. But he does not rest his case on this objection, since the pseudotime narrative and its problems are "peculiar to Cramer's theory, and might well not arise in some other account which employs backward causation" (1994, 199). Rather, since he intends to rule out retrocausal theories more broadly, he rests his case on "a final problem of greater scope which will arise for any stochastic theory of this sort" $(1994,199)$. This is the challenge to which I now turn.

\section{Maudlin's challenge}

Consider the experiment shown schematically in figure 2. A particle is emitted at $\mathrm{S}$ at time $t_{0}$. If it follows the lower path it is detected by absorber A at time $t_{1}$. If it follows the upper path then $\mathrm{A}$ does not detect a particle at $t_{1}$, which triggers an absorber $\mathrm{B}$ to be swung from its initial position behind A to a point on the upper path, where it detects the particle at a later time $t_{2}$. Now what happens if (by means of the beam-splitter BS) a particle is produced in a symmetric superposition of following the lower and upper paths? Standard quantum mechanics tells us that there is a $50 \%$ chance that the particle is detected at $\mathrm{A}$ and absorber $\mathrm{B}$ stays put, and a $50 \%$ chance that absorber B swings round and the particle is detected at B. 
How can the TI account for these statistics? Consider first what happens if one attempts to apply the pseudotime narrative. The puzzle here is that the equiprobability of the two outcomes demands that equal amplitude confirmation waves be received from absorber A and absorber B. But if B sends back a confirmation wave, then it must have been struck by an offer wave, so it must have swung round. And if it has swung round, then the particle is not detected at A. So when a confirmation wave is received from absorber B, the particle must always go to absorber B, despite the fact that the offer-confirmation amplitude product is $1 / 2$. That is, even considered as a useful fiction the pseudotime narrative fails us, since it assigns inconsistent probabilities to one and the same event.

Nor does the TI fare much better if we attempt to apply the standingwave interpretation instead. There is no problem with the standing waves themselves; one consistent solution is a standing wave from $\mathrm{S}$ to A along the lower path, and the other is a standing wave from $\mathrm{S}$ to $\mathrm{B}$ along the upper path. The problem is that the recipe for ascribing probabilities to these outcomes is precisely the inconsistent fragment of the pseudotime narrative just discussed; the particle definitely goes to $\mathrm{B}$ if it has a probability of $1 / 2$ of going to $\mathrm{B}$. The inconsistency arises because the configuration of the absorbers is contingent - the location of $\mathrm{B}$ depends on what happens at A - whereas the TI takes the configuration of absorbers as fixed boundary conditions. As Maudlin notes, the TI recipe for ascribing probabilities assumes that the absorbers "represent a fixed field of possibilities among which the emitter can choose" $(1994,199)$. If the absorbers aren't fixed, the recipe yields inconsistent results.

This difficulty is not limited to the TI, but applies to any theory that incorporates both advanced and retarded causal influences. Just as the retarded causal influences are ill-defined without a fixed configuration of particle sources, so the advanced causal influences are ill-defined without a fixed configuration of particle absorbers. In Maudlin's example, the absorbers move based on earlier events, so no single set of advanced influences can be ascribed to the system. But neither can one ascribe one set of advanced influences at one time and another set of advanced influences at another, on pain of contradiction. Maudlin concludes that "Cramer's theory collapses" (1994, 200), and since "any theory in which both backwards and forwards influences conspire to shape events will face this same challenge" (1994, 201), the entire retrocausal program in quantum mechanics collapses too.

But the TI has not collapsed; a variety of responses have been developed, 
either claiming to accommodate Maudlin's example within the existing TI, or modifying the TI to accommodate it. I run through these responses in the next section, and argue that none of them is satisfactory. The reason, I contend, is that there are two elements to Maudlin's challenge that have not been clearly distinguished. Distinguishing them allows us to evaluate the TI much more clearly; some aspects of it can remain in an adequate theory, while other aspects must be modified. Neither has the retrocausal program in quantum mechanics collapsed; indeed it is flourishing through the work of Price (1994), Sutherland (2008) and Wharton (2010), among others. The evaluation of the TI in light of Maudlin's challenge allows us to judge these alternative retrocausal approaches as well.

\section{Extant responses}

Of the extant responses to Maudlin's challenge, Berkovitz (2002), Kastner (2006) and Marchildon (2006) attempt to accommodate Maudlin's example within the existing TI, and Cramer (2005), Chiatti (2012) and Kastner (2012b, 2013) work with modified versions of the TI. I will start with the former.

Berkovitz (2002) points out that given common views about probabilistic causality, the TI applied to Maudlin's example involves a causal loop, since the confirmation wave returning backwards in time from B is necessary (and sufficient) for the particle to be emitted towards B. Berkovitz then notes that in causal loops the relative frequencies of events can differ significantly from their objective chances. So in this case, the fact that the particle always takes the upper path when B swings round is not inconsistent with the upper path having an objective chance of $1 / 2$. However, it is not clear that Berkovitz intends his analysis as a defense of the TI; he notes that since the link between objective chance and long-run frequency is broken, the TI fails to predict the long-run frequencies of outcomes. Rather than containing an inconsistent recipe for ascribing probabilities to outcomes, the TI contains no such recipe at all. This is not a way forward for the advocate of the TI.

Kastner (2006) identifies the following as a problematic aspect of Maudlin's example; if absorber B does not swing round, then the offer wave on the upper path heads into space, and no confirmation wave is received from the upper path at the source. Typically in the TI, the confirmation waves returning from a complete set of absorbers cancel out to the past of the initial emission 
event. But in this case, the absence of a confirmation wave from the upper path means that the confirmation wave from the lower path propagates into the past, prior to $t_{0}$. On the other hand, if B does swing round, then there is a complete set of absorbers, and the confirmation waves do cancel out prior to $t_{0}$.

The lesson Kastner takes from this is that the emission event is not suitable as a starting-point for the TI analysis, since this event and its past depend on whether B swings round or not, i.e. on the outcome of the experiment. Instead, Kastner takes the offer-confirmation wave pair on the lower path as the starting point of her analysis, since this pair exists whether B swings round or not. The confirmation wave here has an amplitude of $1 / \sqrt{2}$, and so Kastner reasons that the corresponding transaction - in which the particle takes the lower path where it is absorbed by $\mathrm{A}$ - has a probability of $1 / 2$. But the only other possibility is that the particle takes the upper path where it is absorbed by $\mathrm{B}$, so this also must have a probability of $1 / 2$. This recovers the standard quantum mechanical probabilities. However, note that the probability of absorption by $\mathrm{B}$ is obtained by elimination: it must be $1 / 2$ because the probability of absorption by A is $1 / 2$. But Maudlin's challenge is to show how the mechanism of the TI generates these probabilities. Kastner has not explained why a confirmation wave from $\mathrm{B}$ always produces a completed transaction, even though its amplitude is $1 / \sqrt{2}$. Hence Maudlin's challenge has not been addressed.

Marchildon (2006) notes that when B does not swing round, the offer wave along the upper path is still absorbed somewhere; a particle taking this path would be absorbed by something eventually. Due to the retrocausal nature of the pseudotime sequence, it makes no difference how far in the future this absorption event lies; the confirmation wave is still received by the particle source at $t_{0}$. One may as well assume that there is a third absorber $\mathrm{C}$, situated on the upper path beyond the point B swings to. Hence a complete set of confirmation waves is received at the particle source whether or not B swings round, and in either case the confirmation waves cancel out prior to $t_{0}$.

Maudlin's example remains problematic, however; if B does not swing round, the new absorber $C$ returns a confirmation wave with amplitude $1 / \sqrt{2}$, and yet no transaction is ever completed with C. Marchildon attempts to dissolve this problem by appealing to the four-dimensional spacetime implicit in the TI; "the future, though not predictable, is well defined" $(2006,427)$. The four-dimensional block universe is subject to consistency conditions, and 
"in the present case, these conditions are that B absorbs the particle if and only if A does not absorb it" (2006, 427). Given this consistency condition, there are just two possible trajectories, and each is ascribed the probability corresponding to the product of the amplitudes of their respective offer and confirmation waves, i.e. $1 / 2$.

Marchildon is right that there are two possibilities in spacetime; these are the four-dimensional standing waves discussed above. But recall that the ascription of probabilities to these possibilities requires an appeal to a fragment of the pseudotime narrative, even if it is only taken as an instrumental recipe. Marchildon's account retains the inconsistency in this recipe regarding absorber $\mathrm{B}$; absorber $\mathrm{B}$ definitely receives the particle if it has a probability of $1 / 2$ of doing so. Furthermore, his account adds a new inconsistency regarding absorber $\mathrm{C}$; absorber $\mathrm{C}$ definitely does not receive the particle when it has a probability of $1 / 2$ of doing so. The contradiction at the heart of Maudlin's challenge remains.

So maybe there is no way to accommodate Maudlin's example within the TI as it stands; if we want to rescue the theory, perhaps we need to modify the recipe for ascribing probabilities to outcomes. Cramer (2005) attempts to do just that by modifying the way that pseudotime explanations are constructed. In the standard TI, all possible transactions have the same status; what Cramer proposes instead is a hierarchy of possible transactions, ordered by the spacetime interval covered by the transaction. That is, transactions with shorter spacetime interval are given the opportunity to form or not form "before" (in pseudotime) those with longer spacetime interval. This takes care of Maudlin's example, since the possible transaction with absorber A is decided first. The pseudotime sequence now goes like this: The offerconfirmation pair to absorber $\mathrm{A}$ is treated first, and since the product of their amplitudes is $1 / 2$, there is a probability of $1 / 2$ of a transaction forming with A. If it fails to form, then absorber B swings round, and the offerconfirmation pair to absorber B can be considered; it too has an amplitude product of $1 / 2$, corresponding to a probability $1 / 2$ of this transaction forming.

But the hierarchical pseudotime sequence envisioned here is not a general solution to the problem. For one thing, it will not work for Maudlin-type experiments involving photons, since in that case the spacetime interval for every possible transaction will be zero. ${ }^{1}$ For another, it seems to have dif-

\footnotetext{
${ }^{1}$ David Miller made this point during the "TI Cyber-Roundtable" organised by Ruth Kastner (March-August 2011).
} 
ficulty handling interference experiments. Consider a two-path interference experiment (using particles other than photons) in which one path is exactly one wavelength longer than the other. According to Cramer's proposal, one considers the possible transaction along the shorter path first. This transaction has a $50 \%$ chance of forming, and if it forms, then no transaction forms along the longer path. But this is inadequate to the phenomena; it is crucial to the empirical adequacy of the TI that the transaction in interference situations forms along both paths simultaneously. In interference situations, the spacetime interval of the two possible paths has to be irrelevant to transaction formation.

Chiatti (2012) regards the TI pseudotime sequence as superfluous, since he thinks that the structure of transactions can be found within the formalism of standard quantum mechanics. The way to do this, he argues, is to adopt an ontology of particle creation/destruction events; these are the only events that physically exist, and the TI offer and confirmation waves are "a mathematical fiction suited to calculate the statistics of the connection" between a pair of events $(2012,2)$. The physical universe is regarded as a network of particle creation/destruction events, with the TI offer and confirmation waves serving only to coordinate the statistical relations among these events.

Chiatti claims that in this context, Maudlin's argument is unfounded because the two possible transactions have different events at their extremities; absorption by A is a different event than absorption by B. Different extremal events entail different TI-style analyses, and hence we have two simple applications of the theory, not one paradoxical one. This decoupling of the problem fails to show how the event of B-absorption is related to that of Aabsorption. However, Chiatti has a suggestion here, namely that "the event (not $A)^{+}(\text {not } A)^{-}$is itself a transaction termination (null interaction)", so that "the second transaction assumes as its input the output state of the first" (2012, 28). The suggestion is that the event of a particle not being absorbed by A can serve as the terminal event of a transaction, with probability $1 / 2$, and this event can also serve as the initial event of a second transaction that ends with a particle absorbed by B. The trouble with this suggestion is that a null interaction - a particle failing to be destroyed - is not a physical event in Chiatti's ontology, so it is hard to see how it can serve as the terminal event in a transaction. There are not two back-to-back transactions here, but a single transaction with a complex structure.

The most persistent and innovative developer of the TI is Kastner (2010, 
2012a, 2012b, 2013). Kastner suggests that the way to retain the status of pseudotime histories as genuine explanations is to adopt a particular kind of modal realist ontology. Standard TI is most straightforwardly interpreted as entailing that only one of the potential transactions represented by the offer waves exists. Kastner, on the other hand, insists that all the potential transactions exist in a real space of possibilities; she calls the resulting theory "possibilist TI" (PTI). One of these potential transactions becomes actual: when the experiment lies in the future, there are multiple potential transactions between the emission event and the various possible absorbers, but when the experiment lies in the past there is just a single actual completed transaction from the emitter to one of the absorbers. Hence PTI must be embedded within a dynamic A-theory account of time.

Of course, simply reifying the possible transactions does not help address Maudlin's challenge - in fact, it brings his criticism into sharper focus, since the alleged inconsistencies in the pseudotime sequence are now inconsistencies among real events. Rather, Kastner uses her PTI to respond to Maudlin's challenge in two related ways. First, she argues that Maudlin's challenge is no more problematic for the PTI than delayed-choice experiments are for standard quantum mechanics, since the problematic event structure is the same in both cases $(2013,97-101)$. However, this argument only succeeds to the extent that it is agreed that delayed choice experiments are not problematic for standard quantum mechanics. This is far from a foregone conclusion; Cramer notably takes the problems that delayed-choice experiments pose for standard quantum mechanics as one of his motivations for developing the TI (1986, 671). Furthermore, since standard quantum mechanics suffers from the measurement problem, the relevant class of theories with which to compare PTI consists of those that solve the measurement problem-Bohm's theory, the GRW theory and Everettian approaches - and none of these theories is troubled by either delayed-choice experiments or Maudlin's challenge. The fact that PTI is in no worse shape than standard quantum mechanics is not by itself very reassuring.

However, Kastner also has a more direct defense of the PTI from Maudlin's challenge, adapting Wheeler's defense of standard quantum mechanics from the challenge posed by delayed-choice experiments. This strategy is to admit the possibility that the past might be indeterminate in some specified sense. Maudlin assumes that a complete set of confirmation waves at the emitter (i.e. a set whose combined probability is 1 ) is required for the consistency of the theory. Kastner proposes instead that the set of confirmation waves re- 
ceived at the emitter is simply indeterminate, even at times after $t_{0}$ when the emission event lies in the past $(2013,101)$. This is because the confirmation waves depend on the configuration of absorbers, and at times between $t_{0}$ and $t_{1}$ the configuration of absorbers is itself indeterminate. At time $t_{1}$ when the lower offer wave arrives at potential absorber A, a confirmation wave with amplitude $1 / \sqrt{2}$ is returned to the emitter. There is a corresponding chance of $1 / 2$ that this offer-confirmation pair will form a completed transaction to A. If it does not, then absorber B swings into position, a confirmation wave with amplitude $1 / \sqrt{2}$ is returned from this absorber to the emitter as well, and a transaction forms between the emitter and absorber B. In the latter case, Kastner squares the definite formation of a transaction to B with the offer-confirmation amplitude product of $1 / 2$ by arguing that the amplitude product refers to the frequency of this outcome among repeated trials, not to the chance of this very confirmation wave producing a transaction (2013, $96)$.

Kastner's reconceptualization of the TI has certainly advanced the debate on a number of fronts, but the response to Maudlin's challenge is not fully satisfactory. First, the dynamic nature of the possibility space is problematic, even by the standards of A-theories of time. Prior to $t_{1}$, absorption by $\mathrm{B}$ is not a physical possibility (absorber $\mathrm{B}$ receives no offer wave), but after $t_{1}$ absorption by $\mathrm{B}$ becomes a physical possibility. Even if one if prepared to admit that physical possibilities disappear as time marches on (because one of them becomes actual), it is harder to see how physical possibilities can appear; surely if an event at $t_{2}$ is physically possible as of $t_{1}$, it was physically possible as of all earlier times as well. Second, Kastner's interpretation of confirmation wave amplitude seems at odds with her insistence that the realm of future possibilities is "physically efficacious" (2013, 68). It is easy to see how the confirmation wave from $\mathrm{A}$ is physically efficacious: its arrival at the emitter generates the chance of the corresponding transaction forming, where the chance is equal to the amplitude product. But the confirmation wave from $B$ is not physically efficacious in the same way: its arrival does not trigger a chance of $1 / 2$ of the transaction forming, despite the fact that the amplitude product is $1 / 2$. Rather, the transaction always forms on arrival of this confirmation wave, and its amplitude product is to be interpreted as merely an indication of a frequency in a hypothetical ensemble rather than as a causal factor. That is, the role of the confirmation wave in bringing about the completed transaction with the corresponding probability is undermined. 


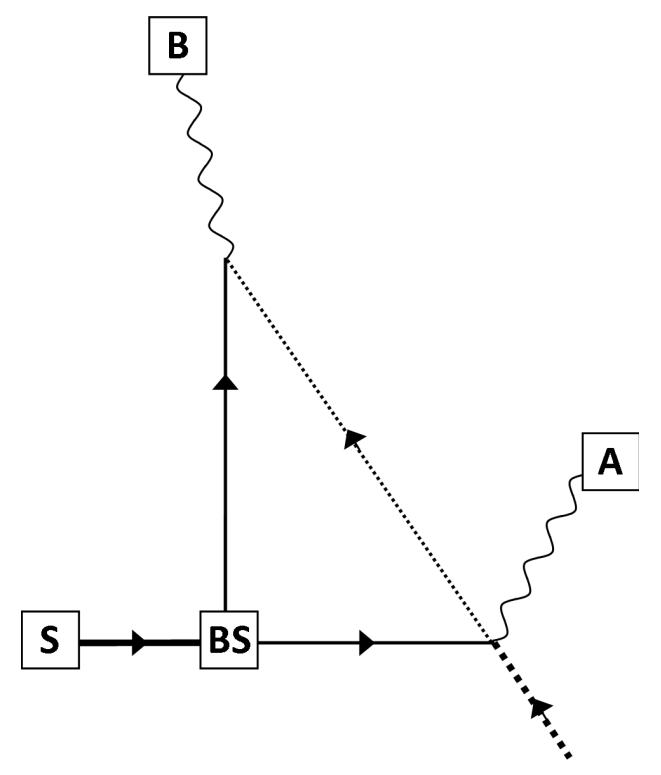

Figure 3: Maudlin's experiment, quantum version.

\section{The first challenge}

It looks like none of the extant responses to Maudlin's challenge are fully adequate. The feature of Maudlin's argument to which all commentators have been attempting to respond is the contingent nature of the absorber structure - the fact that the locations of the absorbers depend on the trajectory of the particle. This is not surprising, since Maudlin himself describes his challenge in these terms: "This picture depends crucially on the idea that the absorbers are somehow just sitting out there in the future, waiting to absorb... But there is no reason for the absorbers to be fixed in the future, unaffected by everything that happens in the present" (1994, 199). However, I think the contingent structure of the absorbers is only one element of Maudlin's challenge, and not the most problematic one.

First, note that Maudlin's experiment involves a mixed quantum/classical system; the particle is a quantum system, but the movable absorber is a classical (macroscopic) system. This, though, looks like an expendable feature of Maudlin's example; one could make all the subsystems quantum mechanical, and still retain the contingent absorber structure. For example, consider the variant of the experiment shown in figure 3 . If a particle is emitted along the 
lower path, it collides with a carefully-timed incoming antiparticle (dotted line) at $t_{1}$. The two particles annihilate, and one of the resulting photons is detected at A. If a particle is emitted along the upper path, then clearly the antiparticle doesn't encounter it at $t_{1}$; instead, the antiparticle travels on, and collides with the particle at a later time $t_{2}$ on the upper path. This time one of the resulting photons is detected at $\mathrm{B}^{2}$

The moving parts in this version of Maudlin's experiment are all quantum systems, but the absorber structure is just as in the original version, with the antiparticle playing the role of the moving absorber. There is a slight difference, namely that in the original version there are two absorbers, one of which moves, whereas in the new version there is just one moving absorber. But this is unimportant; in the original version, it could just as well be absorber A that moves to the upper path when it fails to detect a particle on the lower path. So if it is the contingent absorber structure that is the problematic aspect of Maudlin's example, it ought to be just as problematic in this version. But it does not appear so; one can construct a fairly straightforward pseudotime narrative in this case that yields the right probabilities.

Here is how it goes. A particle and an anti-particle are emitted at the beginning of the experiment, so let us write the initial offer wave as $|O\rangle_{p}|O\rangle_{a}$. The offer wave for the particle is split by the beam-splitter into two equal components, following the lower path and upper path respectively; at this stage, we can write the total offer wave as

$$
\frac{1}{\sqrt{2}}\left(|L\rangle_{p}+|U\rangle_{p}\right)|O\rangle_{a}
$$

The offer wave for the antiparticle travels towards the lower path, where it is split into two terms depending on whether it meets the particle on the lower path or not. That is, the term $|L\rangle_{p}|O\rangle_{a}$ in (1) evolves to $|L\rangle_{p}|L\rangle_{a}$, where

\footnotetext{
${ }^{2}$ In electron-positron annihilation two photons are produced; for simplicity I have only shown one in the diagram. One might object to calling the antiparticle an absorber, since it no longer exists after the absorption event; nevertheless, there are two absorption events with the same contingent structure as in Maudlin's example. Finally, some proponents of the TI insist that absorbers must be macroscopic objects, either because transactions are irreversible (Boisvert and Marchildon 2013, 306) or because only a macroscopic object absorbs with (practical) certainty (Kastner 2013,65). However, since the collapse process is not a literal spacetime process in the TI, there seems little motivation to insist that transactions are irreversible. Similarly, as long as an entity absorbs in this particular experiment, there seems little motivation to insist that it was almost certain to do so.
} 
$|L\rangle_{a}$ is an offer wave for the antiparticle that encounters the particle on the lower path. The term $|U\rangle_{p}|O\rangle_{a}$ in (1) evolves to $|U\rangle_{p}|U\rangle_{a}$, where $|U\rangle_{a}$ is an offer wave for the antiparticle that travels onwards through the lower path and encounters the particle on the upper path. Hence the superposition state (1) as a whole evolves to the "later" (in pseudotime) superposition state

$$
\frac{1}{\sqrt{2}}\left(|L\rangle_{p}|L\rangle_{a}+|U\rangle_{p}|U\rangle_{a}\right) .
$$

Each term returns a confirmation wave from the point at which the particle and antiparticle annihilate; the first term returns a confirmation wave

$\frac{1}{\sqrt{2}}\left\langle\left. L\right|_{p}\left\langle\left. L\right|_{a}\right.\right.$, and the second term returns a confirmation wave $\frac{1}{\sqrt{2}}\left\langle\left. U\right|_{p}\left\langle\left. U\right|_{a}\right.\right.$. Hence the (dual) source receives two confirmation waves, each with amplitude $1 / \sqrt{2}$, and a transaction forms along one of the offer-confirmation pairs with probability $1 / 2$ each. This pseudotime narrative hence successfully ascribes probabilities of $1 / 2$ each to the two possible transactions - the one in which the particles annihilate on the lower path, and the one in which they annihilate on the upper path.

Of course, one still may not want to take the pseudotime narrative literally, since the sequence of events it describes is neither a temporal nor a causal one. The point is that the pseudotime narrative provides a consistent recipe for ascribing probabilities to the two four-dimensional standing waves that are the completed transactions. Hence the TI can account for the standard quantum probabilities in contingent-absorber experiments without any new difficulties or any modifications. Why, then, has Maudlin's example been taken to be so problematic?

\section{The second challenge}

The reason is that the contingent absorber structure in Maudlin's example is not instantiated by quantum systems, but by classical (macroscopic) objects. As explained in the previous section, the straightforward and natural response to the contingent absorber problem just sketched involves incorporating the absorber (the anti-particle) into the TI pseudotime analysis, rather than treating it as part of the environment. But such incorporation is far more problematic if we need to incorporate the state of a macroscopic object into our TI analysis. There are two tricky issues that arise when one contemplates treating a macroscopic object as falling within the scope of a 
TI analysis. The first issue concerns whether the proposed analysis even makes sense; it depends on the way in which TI practitioners conceive of their theory. The second issue concerns the nuts and bolts of constructing such an analysis, given that it makes sense.

To understand the first issue, a little broad-brush history will help. In broad terms, there are two traditions in the interpretation of quantum mechanics. The first, harkening back to Bohr and Heisenberg, takes the distinction between the quantum world and the classical world as basic; call this the Copenhagen tradition. According to this tradition, the world (or at least, the part of it relevant to a given experiment) divides into system and apparatus. The apparatus belongs to the world of experience, and behaves classically. The apparatus delivers results which we take to be produced by an unseen micro-world. Quantum mechanics does not describe the workings of this micro-world directly, but rather describes what we should expect to see when the apparatus interacts with the system. On this view, the system/apparatus divide is built into the theory of quantum mechanics; the observables of the theory correspond to the operations of applying various pieces of macroscopic measuring equipment to the quantum system. Quantum mechanics applies to the interaction between classical measuring devices and quantum systems, and so any attempt to apply quantum mechanics to the measuring equipment itself is fundamentally misguided.

The TI can be understood as part of this Copenhagen tradition - as a way of making it more precise. Emitters and absorbers are part of the classical world; we arrange them in constructing an experiment, and we observe the results they present. TI quantum mechanics generates probabilistic predictions based on the particular arrangement of emitters and absorbers we have constructed. On this understanding of the TI, it is about the interaction of an unseen quantum world with a given arrangement of emitters and absorbers; the arrangement of emitters and absorbers constitutes the environment in which the TI analysis takes place. If we understand the TI in this way, of course, then the straightforward route to resolving Maudlin's challenge is blocked as a matter of principle; there is no way round.

But there is a second tradition in the interpretation of quantum mechanics, stretching from Einstein and Schrödinger through Bell to Maudlin; call this (without too much prejudice, I hope) the realist tradition. According to this tradition, quantum mechanics really does describe the workings of the micro-world, not just system/apparatus interactions. The observables of quantum mechanics should not be conceived in terms of measurement opera- 
tions, but in terms of actual properties of the quantum system. On this view, there is no significant distinction between the micro-world and the macroworld; quantum mechanics applies just as much to the latter as to the former, since macroscopic systems are built out of microscopic ones. Hence quantum mechanics can be applied unproblematically to the measuring devices themselves.

There is no reason in principle why the TI can't be thought of in these terms, and in fact several of those who have developed the TI explicitly do so. Chiatti, for example, writes that "a piece of "common matter" is actually an aggregate of transactions which take place in enormous numbers per second and per cubic centimeter" $(2012,18)$, and Kastner writes that "a macroscopic "observed event" is generally the product of an enormous number of transactions" (2013, 156). Furthermore, there are other retrocausal approaches to quantum mechanics that clearly fall into the realist camp, for example the approaches of Price (1994), Sutherland (2008) and Wharton (2010). If we think of quantum mechanics in this way, the first tricky issue is a non-issue; there is nothing in principle to prevent the incorporation of a macroscopic absorber into a retrocausal analysis.

It is worth noting here that the realist tradition faces a problem that doesn't arise in the Copenhagen tradition; quantum mechanics treats measurements differently from non-measurements (only the former trigger collapse), but if measurements are themselves quantum processes this distinction cannot arise. Various responses to this measurement problem have been developed, and it is interesting that the three major ones all treat Maudlin's experiment by including absorber $\mathrm{B}$ in the system to be analyzed. This is most obvious in the Everett (many-worlds) theory. Applying Everett to Maudlin's original experiment yields the following final state:

$$
\frac{1}{\sqrt{2}}\left(|L\rangle_{p}|Y\rangle_{A}|N, L\rangle_{B}+|U\rangle_{p}|N\rangle_{A}|Y, U\rangle_{B}\right)
$$

where $|Y\rangle_{A}$ and $|N\rangle_{A}$ are states of $\mathrm{A}$ in which it does and does not absorb a particle, and $|N, L\rangle_{B}$ and $|Y, U\rangle_{B}$ are states of B in which it doesn't absorb a particle and remains on the lower branch, and does absorb a particle after swinging to the upper branch. The existence of these two terms, according to the Everettian, explains the observed results; in one branch of reality the particle takes the lower path and is absorbed by A, and in the other branch of reality the particle takes the upper path and absorber B swings round to absorb it there. Bohm's hidden variables theory tells essentially the same 
story, except that one branch is associated with the Bohmian particles, and hence corresponds to the actual result. The GRW collapse theory appeals to the instability of (3) under its collapse dynamics to account for the fact that the final state is (close to) one of the two terms. The lesson (to generalize a little) seems to be that one has to incorporate absorber B into the quantum mechanical analysis if one is to give an adequate realist account of the Maudlin experiment. ${ }^{3}$

As mentioned above, provided that the TI is seen as a direct account of the micro-world in the spirit of the realist tradition, there is nothing to prevent it doing just that. But the details of how this is to be accomplished remain somewhat murky; this is the second tricky issue advertised above. The immediate problem is that TI analysis (as it stands) admits only two kinds of end-points - emission events and absorption events. To be subject to TI analysis, particles must be followed from birth to death. But the particles that make up the absorbers are not emitted at the start of the experiment or absorbed at the end. If we have to know the full life-history of all the particles that make up the absorbers before we can apply this version of the TI, it becomes impossible to apply.

However, the details of the evolution of the particles in the absorbers before and after the experiment seem irrelevant to the analysis at hand. So perhaps we can avail ourselves of the following harmless myth; pretend that all the particles involved in the analysis are created at the beginning of the experiment and destroyed at the end. This myth might be justified by appealing to the fact that wherever and whenever the particles in the absorbers are actually created and destroyed, the offer and confirmation waves over the course of the experiment will correspond to their mythical counterparts. If that justification works, and the myth is adopted, then a pseudotime narrative for Maudlin's original experiment can be given along the lines of the previous section.

Here is how it goes. According to the myth, the test particle and all the particles in the absorbers are emitted at the beginning of the experiment. Let us write the initial offer wave as $|O\rangle_{p}|N\rangle_{A}|N, L\rangle_{B}$ (using the same notation as above), since initially $\mathrm{A}$ and $\mathrm{B}$ have not absorbed a particle and $\mathrm{B}$ is on the

\footnotetext{
${ }^{3}$ Kastner's PTI does not incorporate absorber B into the quantum mechanical analysis - indeed, it cannot do so in principle, since macroscopic objects are systems of completed transactions according to the PTI, and hence cannot appear in quantum superpositions. However, I argued above that Kastner's PTI does not successfully respond to Maudlin's objection.
} 
lower path. The offer wave for the particle is split by the beam-splitter into two equal components, following the lower path and upper path respectively; at this stage, we can write the total offer wave as

$$
\frac{1}{\sqrt{2}}\left(|L\rangle_{p}+|U\rangle_{p}\right)|N\rangle_{A}|N, L\rangle_{B}
$$

The term $|L\rangle_{p}|N\rangle_{A}|N, L\rangle_{B}$ in (4) evolves to $|L\rangle_{p}|Y\rangle_{A}|N, L\rangle_{B}$, and the term $|U\rangle_{p}|N\rangle_{A}|N, L\rangle_{B}$ evolves to $|U\rangle_{p}|N\rangle_{A}|Y, U\rangle_{B}$; hence the superposition state (4) as a whole evolves to the "later" (in pseudotime) superposition state

$$
\frac{1}{\sqrt{2}}\left(|L\rangle_{p}|Y\rangle_{A}|N, L\rangle_{B}+|U\rangle_{p}|N\rangle_{A}|Y, U\rangle_{B}\right) \text {. }
$$

Note that (5) is exactly the same as the Everettian final state (3). Applying the myth again, we assume that the test particle and all the particles in the absorbers are destroyed at this point, so that each term in (5) returns a confirmation wave; the first term returns a confirmation wave $\frac{1}{\sqrt{2}}\left\langle\left. L\right|_{p}\left\langle\left. Y\right|_{A}\left\langle N,\left.L\right|_{B}\right.\right.\right.$,

and the second term returns a confirmation wave $\frac{1}{\sqrt{2}}\left\langle\left. U\right|_{p}\left\langle\left. N\right|_{A}\left\langle Y,\left.U\right|_{B}\right.\right.\right.$. Hence the source receives two confirmation waves, and a transaction forms along one of the offer-confirmation pairs with probability $1 / 2$ each. This pseudotime narrative successfully ascribes probabilities of $1 / 2$ each to the two possible transactions - the one in which the particle takes the lower path and B stays put, and the one in which the particle takes the upper path and B swings round.

As before, one may not want to take this pseudotime narrative literally; it is the fact that the narrative provides a consistent probability assignment to the outcomes that is significant. Taking a realist view of Maudlin's example, one can regard the analysis as describing two possible four-dimensional standing waves, standing waves that include both the particle and absorber $\mathrm{B}$, with the relevant fragment of the pseudotime narrative functioning as a recipe for assigning probabilities to the two possibilities. Furthermore, one can carry this lesson over to retrocausal approaches in general; as long as a given retrocausal approach permits the application of quantum mechanics to the motion of absorber B, Maudlin's challenge is soluble.

\section{Transactions and trajectories}

The analysis just sketched constitutes what I take to be the most natural, and perhaps the only, available solution to Maudlin's challenge (at least among 
block-universe realist accounts). But at what cost have we arrived at this solution? We have had to find a way to incorporate pieces of measuring equipment into a TI-style analysis. Those who regard the TI as a Copenhagen-style interpretation and those who regard it as a dynamic A-theory may object that we have left the TI behind at this point. But whether the account sketched above should count as a version of the TI or a new retrocausal theory is not of central concern; the main issue is whether retrocausal interpretations in general can respond to Maudlin's challenge. Still, even from this broader perspective, some may find the price of incorporating macroscopic objects into the analysis too high, and with good reason.

At issue is the uniqueness of classical trajectories in retrocausal quantum mechanics. It is important to note that TI transactions are not always particle trajectories. Two-slit interference is a case in point; the completed transaction goes through both slits to a point on the screen, so the transaction is not a determinate particle trajectory, but a superposition of such trajectories. In fact, this is a generic feature of interference; whenever two or more distinct offer waves contribute to the amplitude at the absorption point, the resulting transaction incorporates the trajectories corresponding to all the offer waves to that point. So the TI-style theories do not always recover determinate particle trajectories in the way that, for example, Bohm's theory does. But provided such indeterminacy is kept confined to the microworld, this is arguably not a problem.

The worry about incorporating macroscopic objects into a TI-style analysis is that it opens the door for macroscopic objects to have indeterminate trajectories. Thanks to decoherence, interference effects are tiny for macroscopic objects, but they do not go away entirely. So in my treatment of the Maudlin experiment above, the amplitudes of the terms in (5) are affected by the presence of anomalous interference terms. For example, there are lowamplitude terms in which the particle takes the lower path, tunnels through absorber A without triggering it, and then veers over to the upper path to be absorbed by B. So in reality, the wave amplitude at the upper-path location for B includes tiny contributions from offer waves that have not travelled via the upper path. According to the TI, then, the completed transaction contains a large contribution following the standard trajectory, but also minor contributions following the anomalous trajectories. This might seem to threaten the determinacy of trajectories for macroscopic objects, either rendering the TI empirically inadequate or turning it into a baroque version of Everett. 
But perhaps such worries can be deflected; the additional terms are, after all, very small. One might quite reasonably insist that a transaction in which one trajectory is so dominant simply is, for all practical purposes, that trajectory. Provided that no significant interference occurs involving macroscopic objects, the determinacy of macro-trajectories is (arguably) safe. However, there is a further worry that cannot be dealt with in this way. If the TI is interpreted in the realist tradition, there is nothing to prevent it being applied to the state of the universe as a whole (just as Everett, Bohm and GRW can be so applied). Indeed, this is one of the touted advantages of the realist tradition. We wouldn't need the myth for such an application; we really would be following every particle from birth to death. But there is no guarantee that interference between macroscopically distinct terms can be suppressed in such an application; it depends on the global structure of the universe. For example, in highly symmetric universes, branching in the initial stages of the universe might be matched by "reverse branching" (i.e. interference) in the final stages. In that case, there would be no unique trajectory corresponding to the evolution of the macroscopic objects in the universe, and again the TI would either be empirically inadequate or reduce to a version of Everett.

How far do these worries carry over to other retrocausal approaches? In large part, the answer depends on the underlying ontology of the theory concerned. Some retrocausal approaches, such as that of Wharton (2010), are similar to the TI in that systems are modelled in terms of waves. So as in the TI, we would need some assurance that the waves that represent macroscopic objects are always well localized, and this might ultimately depend on cosmology. Other retrocausal approaches, such as that of Price (1994), model systems entirely in terms of the properties of localized particles. Such approaches are clearly immune to the concerns raised above, since all objects are always well localized in such models. ${ }^{4}$ A third set of retrocausal approaches, such as the retrocausal Bohmian approach of Sutherland (2008), models systems in terms localized particles that are guided by waves. These approaches are also immune to the concerns raised above, since even if the global wavefunction of the universe is a superposition of macroscopically distinct terms, the particles will all be associated with one of these terms.

These non-TI retrocausal approaches are all in their infancy, and time

\footnotetext{
${ }^{4}$ See also Price and Wharton (2013), which is non-commital between a wave and a particle ontology.
} 
will tell if the general strategy proves fruitful in understanding quantum mechanics. The main point I hope to have defended here is that for any such approach, provided it can be understood within the realist tradition in quantum mechanics, and provided it can be applied to model the trajectories of macroscopic objects, Maudlin's challenge is not fatal.

\section{Acknowledgments}

I would like to thank the participants in the "TI Cyber-Roundtable" (March-August 2011) for illuminating discussions on many aspects of the TI, and especially Ruth Kastner for organizing and moderating the event and helpful discussions of PTI. I also acknowledge the useful comments of several reviewers for this journal.

\section{References}

Berkovitz, J. (2002), "On causal loops in the quantum realm", in T. Placek and J. Butterfield (eds.), Non-locality and Modality. Dordrecht: Kluwer, $235-257$.

Boisvert, J.-S. and L. Marchildon (2013), "Absorbers in the transactional interpretation of quantum mechanics", Foundations of Physics 43: 294309.

Chiatti, L. (2012), "The transaction as a quantum concept", arXiv:1204.6636.

Cramer, J. G. (1986), "The transactional Interpretation of quantum mechanics", Reviews of Modern Physics 58: 647-687.

- (2005), "The quantum handshake: a review of the transactional interpretation of quantum mechanics", presented at Time-Symmetry in Quantum Mechanics conference, Sydney, Australia, July 23, 2005. Available at: http://faculty.washington.edu/jcramer/PowerPoint/Syd ney_20050723_a.ppt

Deutsch, David (1999), "Quantum theory of probability and decisions", Proceedings of the Royal Society of London A455: 3129-3137. 
Kastner, R. E. (2006), "Cramer's transactional interpretation and causal loop problems", Synthese 150: 1-14.

- (2010), "The quantum liar experiment in Cramer's transactional interpretation", Studies in History and Philosophy of Modern Physics 41: 86-92.

_ (2012a), "The possibilist transactional interpretation and relativity", Foundations of Physics 42: 1094-1113.

(2012b), "On delayed choice and contingent absorber experiments", ISRN Mathematical Physics vol. 2012: article ID 617291.

- (2013), The Transactional Interpretation of Quantum Mechanics. Cambridge: Cambridge University Press.

Marchildon, L. (2006), "Causal loops and collapse in the transactional interpretation of quantum mechanics", Physics Essays 19: 422-429.

Maudlin, T. (1994), Quantum Non-Locality and Relativity. Oxford: Blackwell.

Price, H. (1994), "A neglected route to realism about quantum mechanics", Mind 103: 303-336.

Price, H. and K. Wharton (2013), "Dispelling the quantum spooks - a clue that Einstein missed?", arXiv:1307.7744.

Sutherland, R. I. (2008), "Causally symmetric Bohm model", Studies In History and Philosophy of Modern Physics 39: 782-805.

Wharton, K. (2010), "A novel interpretation of the Klein-Gordon equation", Foundations of Physics 40: 313-332. 